\title{
Association between a relative afferent pupillary defect using pupillography and inner retinal atrophy in optic nerve disease
}

This article was published in the following Dove Press journal:

Clinical Ophthalmology

9 October 2015

Number of times this article has been viewed

\author{
Go Takizawa' \\ Atsushi Miki ${ }^{1-3}$ \\ Fumiatsu Maeda ${ }^{4}$ \\ Katsutoshi Goto' \\ Syunsuke Araki' \\ Yoshiaki leki' \\ Junichi Kiryu' \\ Kiyoshi Yaoeda ${ }^{3,5}$ \\ 'Department of Ophthalmology, \\ Kawasaki Medical School, \\ ${ }^{2}$ Department of Sensory Science, \\ Faculty of Health Science and \\ Technology, Kawasaki University of \\ Medical Welfare, Kurashiki, Okayama, \\ ${ }^{3}$ Division of Ophthalmology and Visual \\ Sciences, Niigata University Graduate \\ School of Medical and Dental \\ Sciences, Niigata, Japan; ${ }^{4}$ Department \\ of Orthoptics and Visual Sciences, \\ Faculty of Medical Technology, Niigata \\ University of Health and Welfare, \\ Niigata, Japan; ${ }^{5}$ Yaoeda Eye Clinic, \\ Nagaoka, Japan
}

Purpose: The aim of this study was to compare the asymmetrical light reflex of the control subjects and patients with optic nerve disease and to evaluate the relationships among the relative afferent pupillary defect (RAPD), visual acuity (VA), central critical fusion frequency (CFF), ganglion cell complex thickness (GCCT), and circumpapillary retinal nerve fiber layer thickness (cpRNFLT) using spectral-domain optical coherence tomography.

Materials and methods: Using a pupillography device, the RAPD scores from 15 patients with unilateral optic nerve disease and 35 control subjects were compared. The diagnostic accuracy of the RAPD amplitude and latency scores was compared using the area under the receiver operating characteristic curve. Thereafter, we assessed the relationships among the RAPD scores, VA, central CFF, GCCT, and cpRNFLT.

Results: The average RAPD amplitude score in patients with optic nerve disease was significantly higher than that of the control subjects $(P<0.001)$. The average RAPD latency score in patients with optic nerve disease was significantly higher than that of the control subjects $(P=0.001)$. The area under the receiver operating characteristic curve for the RAPD amplitude score was significantly higher than that for the latency score $(P=0.010)$. The correlation coefficients for the RAPD amplitude and latency scores were $0.847(P<0.001)$ and $0.874(P<0.001)$ for VA, -0.868 $(P<0.001)$ and $-0.896(P<0.001)$ for central CFF, $-0.593(P=0.020)$ and $-0.540(P=0.038)$ for GCCT, and $-0.267(P=0.337)$ and $-0.228(P=0.413)$ for cpRNFLT, respectively.

Conclusion: Our results suggest that pupillography is useful for detecting optic nerve disease.

Keywords: circumpapillary retinal nerve fiber layer, ganglion cell complex, central critical fusion frequency, visual acuity, spectral-domain optical coherence tomography

\section{Introduction}

The swinging flashlight test ${ }^{1,2}$ has widely been used in the clinical setting as a simple and easy method for detecting a relative afferent pupillary defect (RAPD). ${ }^{3-5}$ The quantitative evaluation of the RAPD is performed using a neutral density (ND) filter, ${ }^{6}$ and this method is applied for the diagnosis and observation of optic nerve disease. However, the quantification of the RAPD using an ND filter may cause a discrepancy

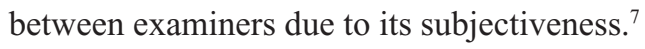

Recently, a new automated pupillography device has become commercially available. The device measures the pupillary light reflex by alternating stimulation to both eyes and can calculate two kinds of RAPD scores from the pupillary constriction amplitude and latency. ${ }^{8-11}$ This machine keeps a fixed duration, interval, and intensity of stimulation and can therefore measure a stable light reflex. The method using the conventional ND filter determines the RAPD by weakening the stimulation intensity
Department of Ophthalmology, Kawasaki Medical School, 577 Matsushima, Kurashiki, Okayama 70I-0192, Japan

Tel $+8 \mid 86462$ IIII

Fax $+8 \mid 86464$ I565

Email amiki@tc5.so-net.ne.jp 
used for the unaffected eye until the reflexes from each eye become equal. On the other hand, the pupillography device calculates the RAPD based on a ratio of the amplitude and latency of the light reflex of each eye induced by constant stimulation luminance.

The relationship between the RAPD determined using an ND filter and the visual function or ocular morphology in patients with optic nerve disease has been reported. ${ }^{12}$ In more recent studies, thinning of the inner retinal thickness and the circumpapillary retinal nerve fiber layer thickness (cpRNFLT) in patients with optic nerve disease has also been demonstrated using spectral-domain optical coherence tomography (SD-OCT). ${ }^{13}$

There have been several studies comparing the RAPD using an ND filter and the retinal thickness in optic nerve disease and glaucoma. ${ }^{14-16}$ Only a few previous reports have examined the correlations between the scores measured with the pupillography device, the visual function, and retinal thickness in glaucoma. ${ }^{8,10,11}$ However, to the best of our knowledge, there is no report comparing the RAPD using automated pupillography and the retinal thickness in optic nerve diseases other than glaucoma.

In the present study, we examined and compared the RAPD scores using pupillography, the visual acuity (VA), central critical fusion frequency (CFF) score, ganglion cell complex thickness (GCCT), and cpRNFLT in patients with unilateral or asymmetrical optic nerve disease.

\section{Materials and methods Study enrollment}

We examined consecutive patients with optic nerve diseases and control subjects treated between November 2012 and December 2013. This study adhered to the tenets of the Declaration of Helsinki and was approved by the institutional review board committee of Kawasaki Medical School. Informed consent for examinations was obtained from all patients. We included patients with unilateral or asymmetrical optic nerve diseases and patients presenting with $\mathrm{VA}>0.05 \log \mathrm{MAR}$ in the unaffected eye. These patients with optic nerve disease underwent an examination of the VA, central CFF, visual field, anterior segment, and ocular fundus. We excluded patients with retinal disease, glaucoma, or a history of intraocular surgery. We also enrolled control subjects without any eye issues besides refractive error.

\section{Pupillography and waveform analysis}

We used the RAPDx ${ }^{\circledR}$ instrument (Konan Medical USA, Inc., Irvine, CA, USA) to record and analyze the pupillary light reflex. The pupillography device measurements were taken in a dark room. We performed the measurements after a dark adaptation of approximately 5 minutes before testing. The measurements comprised alternating stimulation of both eyes and the pupil movement of both eyes was simultaneously recorded. The position was adjusted such that the subject places his or her forehead on the forehead plate of the device, and a pupil was reflected in the monitor. This machine has an LCD monitor for the right and left eyes and a built-in lens to focus. A plate to partition off the right and left eyes is present in the center and enables the stimulation of only one eye at a time. We presented a green object as a fixation target for each eye that was adjusted depending on the position of eyes.

The intensity of the stimulus light was $384 \mathrm{~cd} / \mathrm{m}^{2}$, the stimulus color was white, and the area of stimulation was $30^{\circ}$ of the visual field. The duration of stimulation was 0.2 second, and the presentation interval of the stimulation was 2.1 seconds. The stimulation was presented 18 times. The background illuminance was $0.01 \mathrm{~cd} / \mathrm{m}^{2}$. The amplitude was defined as the ratio of the maximum change in the pupil size divided by the resting pupil size. The RAPD amplitude score was defined by $10 \times \log _{10}$ (amplitude of the right eye/ amplitude of the left eye). The latency was the interval between the beginning of the stimulus and the detection of a significant pupil velocity, which was considered the onset of the pupil light reflex. The RAPD latency score was defined by $10 \times \log _{10}$ (latency of the right eye/latency of the left eye).

\section{Optical coherence tomography}

In all patients, ganglion cell complex (GCC) and circumpapillary retinal nerve fiber layer (cpRNFL) images were obtained by RTVue-100 SD-OCT (Optovue Inc., Fremont, CA, USA), which acquires 26,000 A-scans per second and provides a $5 \mu \mathrm{m}$ depth resolution in tissue.

The GCC protocol consists of one horizontal line scan $7 \mathrm{~mm}$ in length (467 A-scans) and 15 vertical line scans $7 \mathrm{~mm}$ in length (each $400 \mathrm{~A}$-scans) at $0.5 \mathrm{~mm}$ intervals; the center of the GCC scan is shifted $0.75 \mathrm{~mm}$ temporally to improve the sampling of the temporal periphery. This scan configuration provides $14,810 \mathrm{~A}$-scans in 0.58 second. The GCCT is measured from the inner limiting membrane to the outer edge of the inner plexiform layer.

The cpRNFLTs were determined by the nerve head map $4 \mathrm{~mm}$ protocols in the three-dimensional baseline mode, in which the data along a $3.45 \mathrm{~mm}$ diameter circle around the optic disc were recalculated with a map created from face 
imaging that used six circular scans ranging from $2.5 \mathrm{~mm}$ to $4.0 \mathrm{~mm}$ in diameter (587 or $775 \mathrm{~A}$-scans each) centered on the optic disc, and 12 linear radial scan data inputs $(3.4 \mathrm{~mm}$ length, $452 \mathrm{~A}$-scans each). This scan protocol provides 9,510 A-scans in 0.39 second.

\section{Statistical analysis}

Statistical analyses were performed with the SPSS 20 software program (SPSS, Inc., Chicago, IL, USA) and MedCalc version 11 software program (MedCalc Software bvba, Mariakerke, Belgium). The differences between patients with optic nerve disease and the control subjects were compared by an analysis of covariance using age as a covariate. To investigate the ability of the RAPD amplitude and latency scores to differentiate patients with optic nerve disease from the control subjects, receiver operating characteristic (ROC) curves of the RAPD score were drawn. The area under the ROC curve (AUC) was calculated for each RAPD score.

Using Pearson's correlation coefficient and linear regression, the relationships among the RAPD amplitude score, RAPD latency score, VA, central CFF, GCCT, and cpRNFLT were graphically examined. A value of $P<0.05$ was considered to be statistically significant. We calculated the $95 \%$ confidence interval $(95 \% \mathrm{CI})$ from the RAPD of the control subjects. When the RAPD score of patients with optic nerve disease was outside the $95 \% \mathrm{CI}$, the score was considered abnormal.

\section{Results}

Fifty subjects ( 15 patients with optic nerve disease and 35 control subjects) were enrolled in the study. The mean age was $51.4 \pm 20.2$ years (range, 11-75 years) for patients with optic nerve disease and $23.3 \pm 5.9$ years (range, 6-36 years) for controls. The affected side was the right eye in six patients in the optic nerve disease group and the left eye in the other nine patients. Patients with optic neuritis included one patient with papillitis, three with retrobulbar optic neuritis, four with optic atrophy with an unclear history, and two with multiple sclerosis (MS). Tables 1 and 2 summarize the characteristics and the examination results of patients with optic nerve disease. One patient was excluded because she could not be adequately evaluated due to frequent blinking.

The control subjects were significantly younger than patients with optic nerve disease $(P<0.001)$. For that reason, the differences between patients with optic nerve disease and the control subjects were compared by an analysis of covariance using age as a covariate.
Table I Clinical characteristics of the 15 patients with optic nerve disease $(n=15)$

\begin{tabular}{|c|c|}
\hline Age (years) & $51.4 \pm 20.15(11-75)$ \\
\hline Female, n (\%) & $7(46.6)$ \\
\hline Duration (months) & $\begin{array}{l}85.1 I \pm I 74.42(0.03-600 \\
4 \text { patients undefined })\end{array}$ \\
\hline \multicolumn{2}{|l|}{ Refractive error (diopter) } \\
\hline Average of the two eye & $-0.39| \pm 0.74|(0-4.00)$ \\
\hline Between-eye absolute differences & 0.017 \\
\hline \multicolumn{2}{|l|}{ Visual acuity (logMAR) } \\
\hline Average of the two eye & $0.30 I \pm 0.685(2.00$ to -0.18$)$ \\
\hline Between-eye absolute differences & 0.077 \\
\hline \multicolumn{2}{|l|}{ Central CFF (Hz) } \\
\hline Average of the two eye & $30.05 \pm I 1.74(0-46)$ \\
\hline Between-eye absolute differences & 4.167 \\
\hline \multicolumn{2}{|l|}{ GCCT $(\mu \mathrm{m})$} \\
\hline Average of the two eye & $82.53 \pm 13.13(57.55-100.34)$ \\
\hline Between-eye absolute differences & 4.787 \\
\hline \multicolumn{2}{|l|}{$\operatorname{cpRNFLT}(\mu \mathrm{m})$} \\
\hline Average of the two eye & $90.15 \pm 22.69(56.67-152.4 I)$ \\
\hline Between-eye absolute differences & 5.756 \\
\hline
\end{tabular}

Notes: Data are mean \pm SD (range).

Abbreviations: SD, standard deviation; CFF, critical fusion frequency; GCCT, ganglion cell complex thickness; cpRNFLT, circumpapillary retinal nerve fiber layer thickness.

\section{Comparison between patients with optic nerve disease and the control subjects}

The modulus median of the RAPD amplitude score was $1.910 \pm 1.408 \log$ units in patients with optic nerve disease, and was significantly higher than that of the control subjects ( $0.050 \pm 0.077 \log$ units $)(P<0.001)$. The modulus median of the RAPD latency score was $0.360 \pm 0.325 \log$ units in patients with optic nerve disease, and was significantly higher than that of the control subjects $(0.080 \pm 0.071 \log$ units $)(P=0.001)$ (Figure 1).

The mean RAPD amplitude score of the control subjects was $0.016 \pm 0.111 \mathrm{log}$ units (range, from -0.24 to $0.24 \mathrm{log}$ units), and the $95 \%$ CI ranged from -0.022 to 0.054 . The mean RAPD amplitude score of patients with optic nerve disease was $0.253 \pm 2.670 \log$ units (range, from -4.02 to $5.67 \log$ units), and all of the amplitude scores from the patients were outside the $95 \% \mathrm{CI}$. The mean RAPD latency score of the control subjects was $-0.010 \pm 0.118 \log$ units (range, from -0.24 to $0.22 \mathrm{log}$ units), and the $95 \% \mathrm{CI}$ ranged from -0.051 to 0.030 . The mean RAPD latency score of patients with optic nerve disease was $-0.052 \pm 0.511$ log units (range, from -1.23 to $0.84 \log$ units). Regarding the RAPD latency score, 13 patients were outside the $95 \% \mathrm{CI}$, and two patients were within the $95 \%$ CI. 


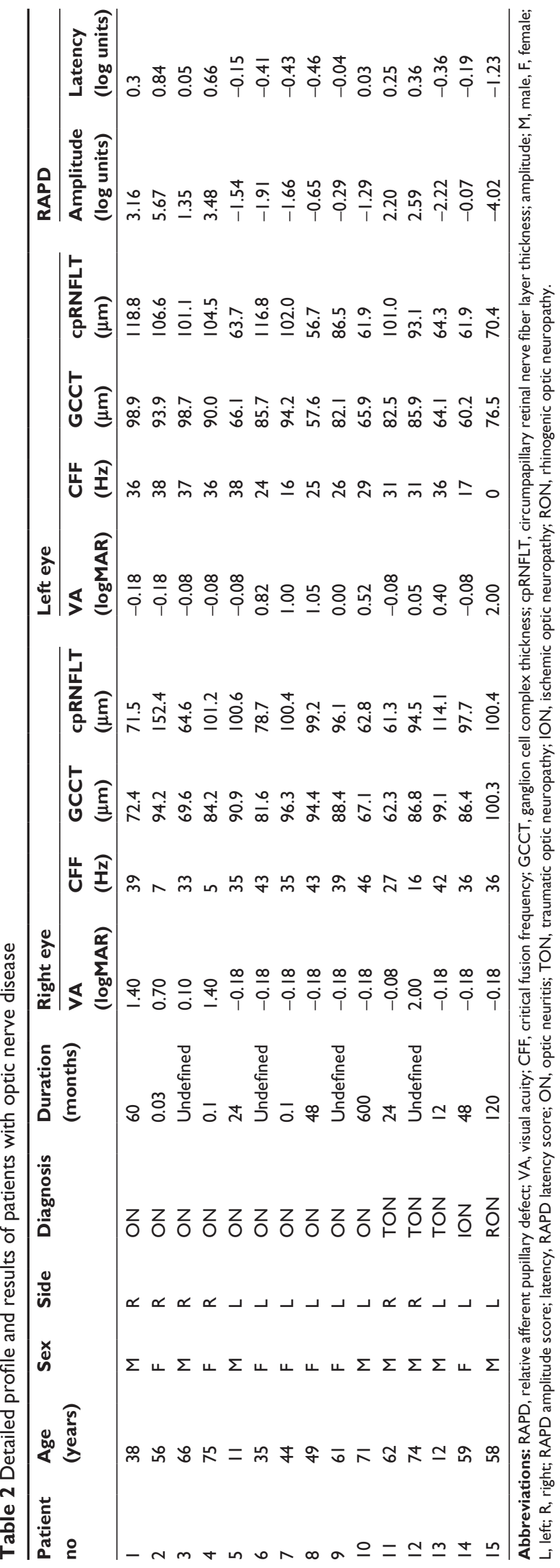

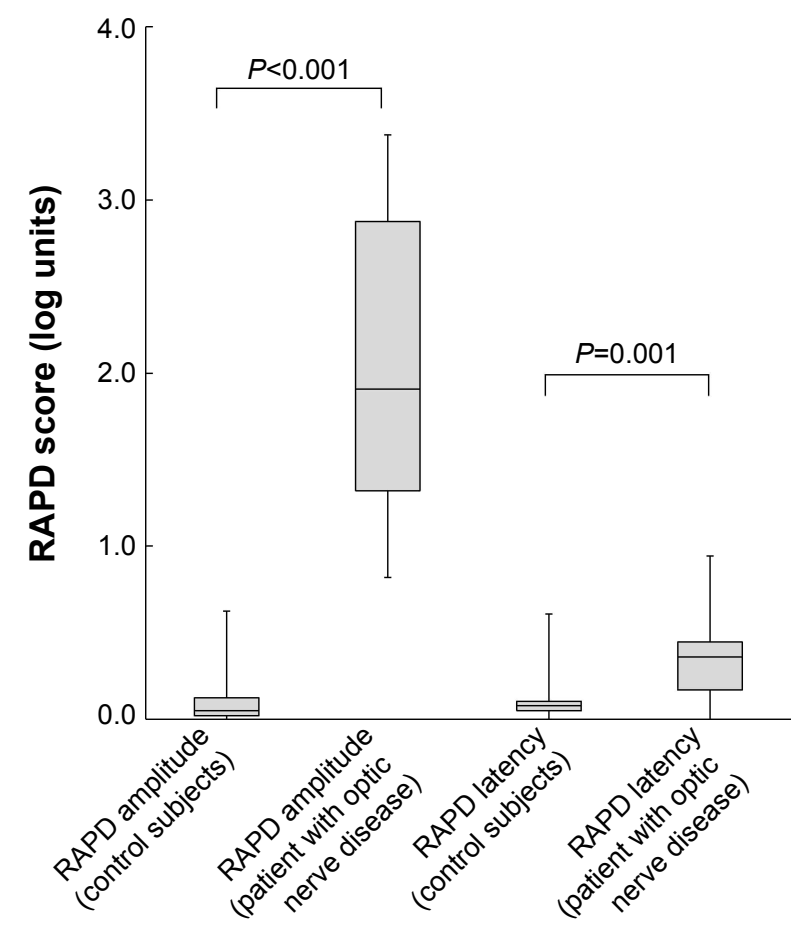

Figure I The relative afferent pupillary defect (RAPD) scores of the optic nerve disease group and the control group.

Note: The RAPD amplitude and latency scores of the optic nerve disease group were significantly higher than that of the control group $(P<0.001$ and $P=0.00$ I, respectively).

The ROC curves of the RAPD amplitude score and latency score are shown in Figure 2. The AUC of the RAPD amplitude score (1.000) was significantly higher than that of the latency score $(0.812)(P=0.010)$.

\section{Relationships between the RAPD score and the VA, central CFF, GCCT, and cPRNFLT of patients with optic nerve disease}

The correlation coefficient between the RAPD amplitude score and the VA of patients with optic nerve disease was $R=0.847(P<0.001)$. Five patients (patient nos 3, 5, 9, 11, and $14)$ in whom the laterality of the VA was small (|difference of VA $\leq 0.18 \log$ MAR) showed a RAPD amplitude score outside the $95 \%$ CI. The correlation coefficient between the RAPD latency score and the VA of patients with optic nerve disease was $R=0.874(P<0.001)$ (Figure 3).

The correlation coefficient between the RAPD amplitude score and central CFF of patients with optic nerve disease was $R=-0.868(P<0.001)$. Five patients (patient nos 1,3 , 5,11 , and 13) in whom the laterality of the central CFF was small ( $\mid$ difference of central CFF $\mid \leq 6 \mathrm{~Hz}$ ) showed a RAPD amplitude score outside the $95 \%$ CI. The correlation 


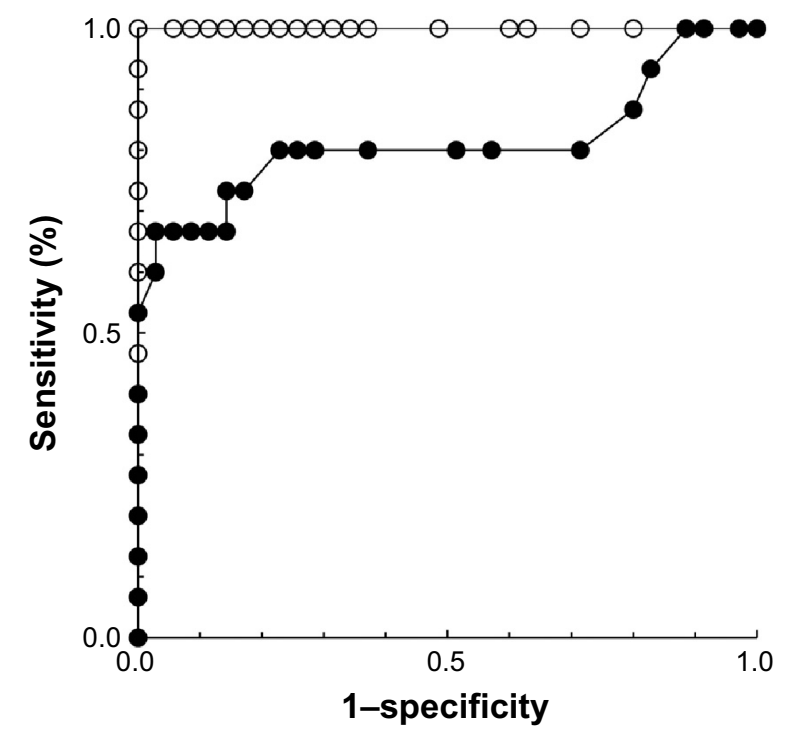

Figure $\mathbf{2}$ The receiver operating characteristic (ROC) curves of the relative afferent pupillary defect (RAPD) amplitude and latency scores.

Notes: Open circles show the RAPD amplitude score, and closed circles show the RAPD latency score. The area under the ROC curves (AUC) of the amplitude score was significantly higher than that of the latency score $(P=0.010)$. AUC: RAPD amplitude score: 1.000 (95\% confidence interval [Cl]: 0.929-I.000), RAPD latency score: 0.812 (95\% Cl: 0.677-0.909).

coefficient between the RAPD latency score and the central CFF of patients with optic nerve disease was $R=-0.896$ $(P<0.001)$ (Figure 4).

The correlation coefficient between the RAPD amplitude score and GCCT of patients with optic nerve disease was $R=-0.593$ ( $P=0.020$ ). Four patients (patient nos $2,7,10$, and 12 ) in whom the laterality of the GCCT was small (difference of GCCT $\mid \leq 2.1 \mu \mathrm{m}$ ) showed a RAPD amplitude score outside the $95 \%$ CI. The correlation coefficient between the RAPD latency score and the GCCT of patients with optic nerve disease was $R=-0.540$ ( $P=0.038$ ) (Figure 5).

The correlation coefficient between the RAPD amplitude score and cpRNFLT of patients with optic nerve disease was $R=-0.267(P=0.337)$. Four patients (patient nos 4, 7, 10, and 12 ) in whom the laterality of the cpRNFLT was small (|difference of cpRNFLT $\mid \leq 3.3 \mu \mathrm{m}$ ) showed a RAPD amplitude score outside the $95 \% \mathrm{CI}$. The correlation coefficient between the RAPD latency score and the cpRNFLT of patients with optic nerve disease was $R=-0.228(P=0.413)$ (Figure 6).

\section{Discussion}

Comparison between patients with optic nerve disease and the control subjects

The RAPD amplitude scores measured by this method deviated from the $95 \% \mathrm{CI}$ and correctly detected the affected eye in all patients with optic nerve disease. The method used to measure the conventional RAPD uses an ND filter to weaken the stimulation intensity for the unaffected eye and is widely used in the clinical setting. In contrast, the pupillography device calculates the RAPD scores from the pupil movement induced by stimulation with light of constant intensity, while the duration and interval time are set constant. In addition, this device includes an automatic eye-tracking function. Therefore, this method should be able to measure the exact RAPD.
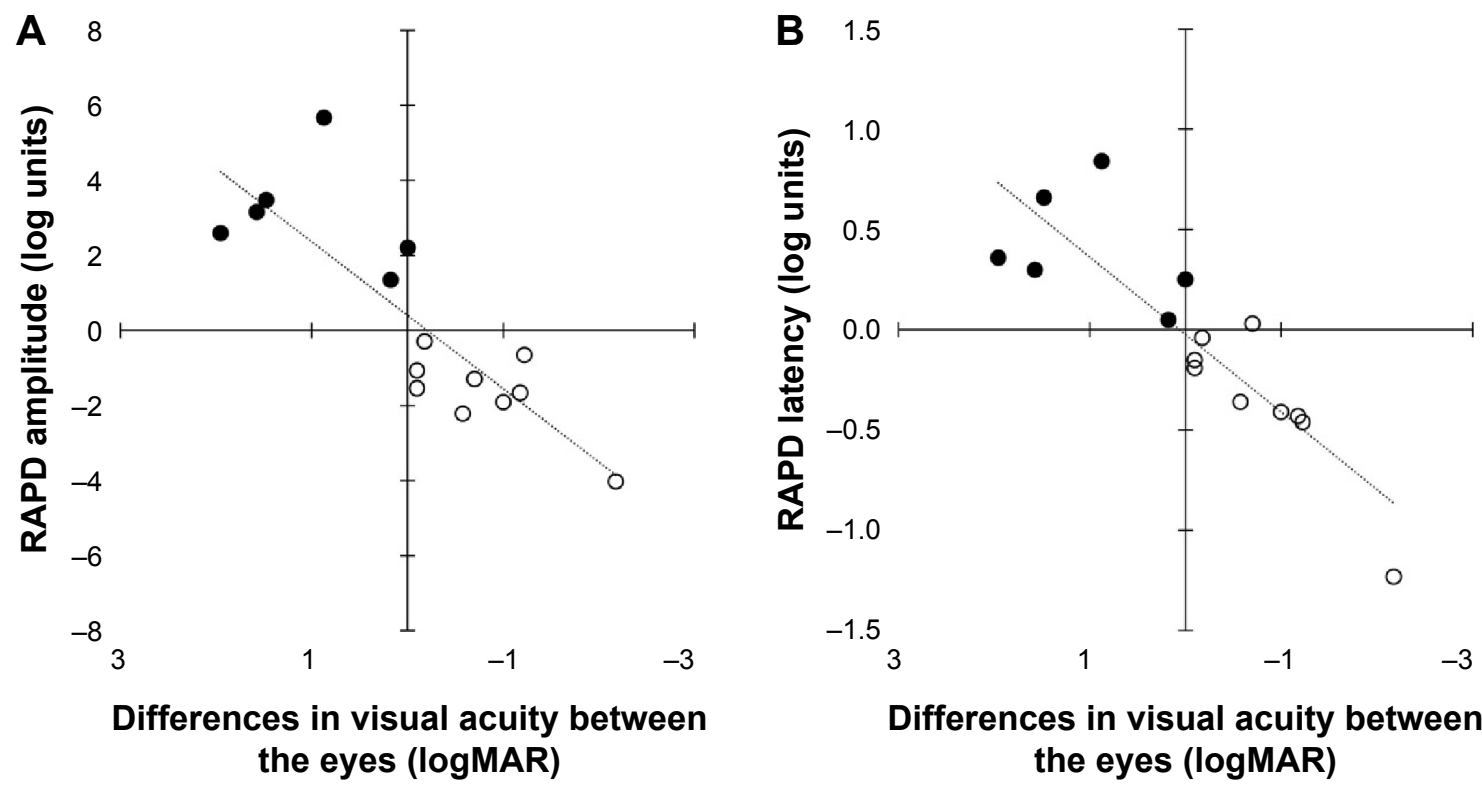

Differences in visual acuity between
the eyes (logMAR)

Figure 3 The relationship between the relative afferent pupillary defect (RAPD) and differences in visual acuity (VA).

Notes: Closed circles show patients in whom the right side was affected, and open circles show patients with left optic neuropathy. $R A P D$ amplitude score: $R=0.847$, $P<0.00$ I (A), RAPD latency score: $R=0.874, P<0.00$ I (B). 

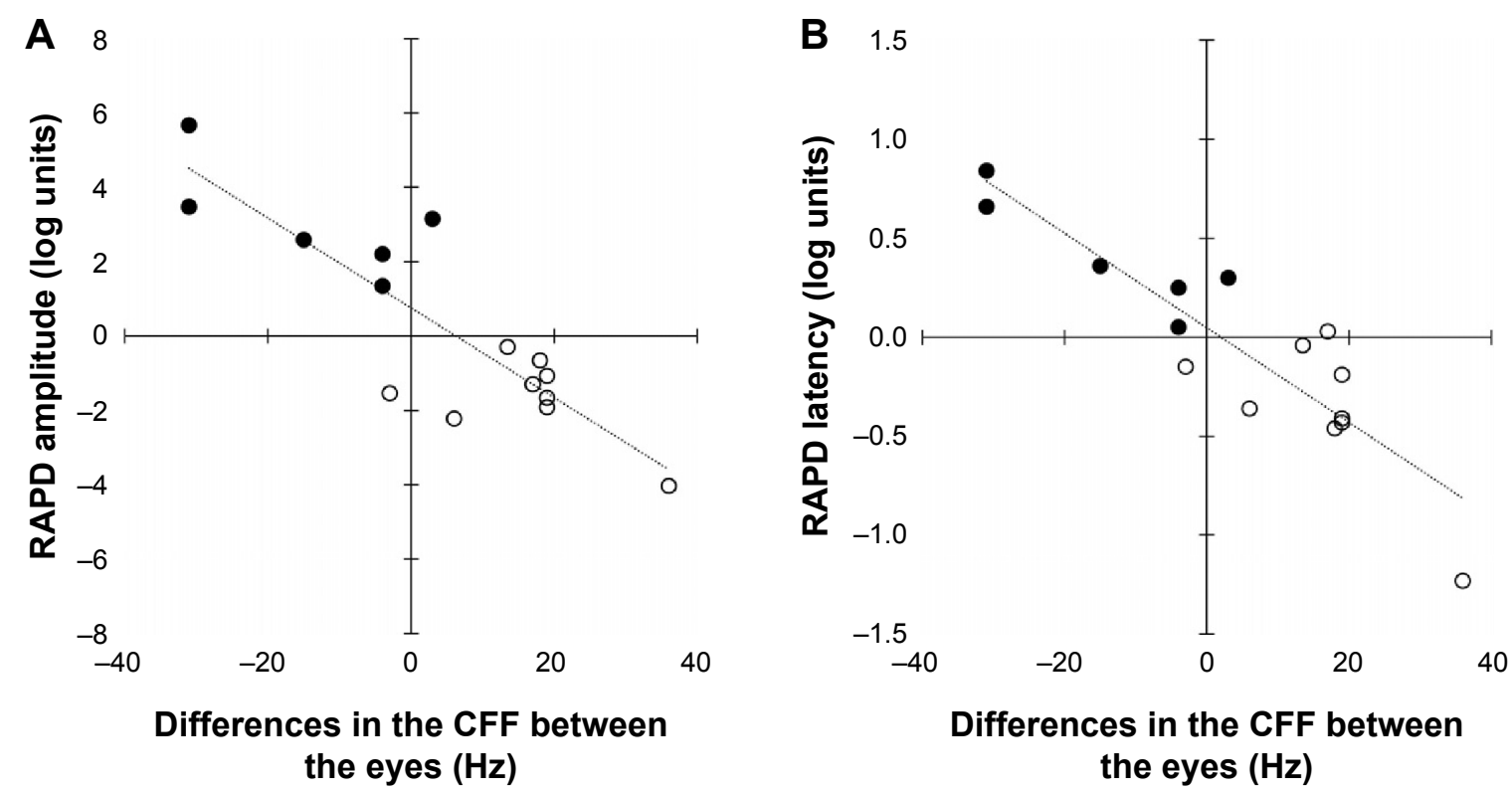

Figure 4 The relationship between the relative afferent pupillary defect (RAPD) and differences in the central critical fusion frequency (CFF).

Notes: Closed circles show patients in whom the right side was affected, and open circles show patients with left optic neuropathy. RAPD amplitude score: $R=-0.868$, $P<0.00$ I (A), RAPD latency score: $R=-0.896, P<0.00$ I (B).

The AUC of the RAPD latency score was significantly lower than the amplitude score in patients with optic nerve disease. The light reflex latency in patients with optic nerve disease has been previously reported. ${ }^{17-19}$ Lowenstein was the first to explain the prolongation of the latency with "low intensity reactions". ${ }^{17}$ Ellis subsequently explained that the latency is influenced by stimulation intensity, ${ }^{19}$ which extends the latency so that the amplitude becomes low. Recently, Chang et al reported that the pupil is expected to slowly constrict and dilate whenever the response is small. ${ }^{20}$ The latency has been thought to correlate with the amplitude according to these previous reports. Therefore, the VA, central CFF, and GCCT, which correlated with the RAPD amplitude score, should also be correlated with the RAPD latency score. In this study, the stimulus presentation interval was constant. Therefore, the pupil diameter before the stimulation might have been unstable. Therefore, the measurements of the RAPD latency score may be inaccurate when the hippus intervenes.

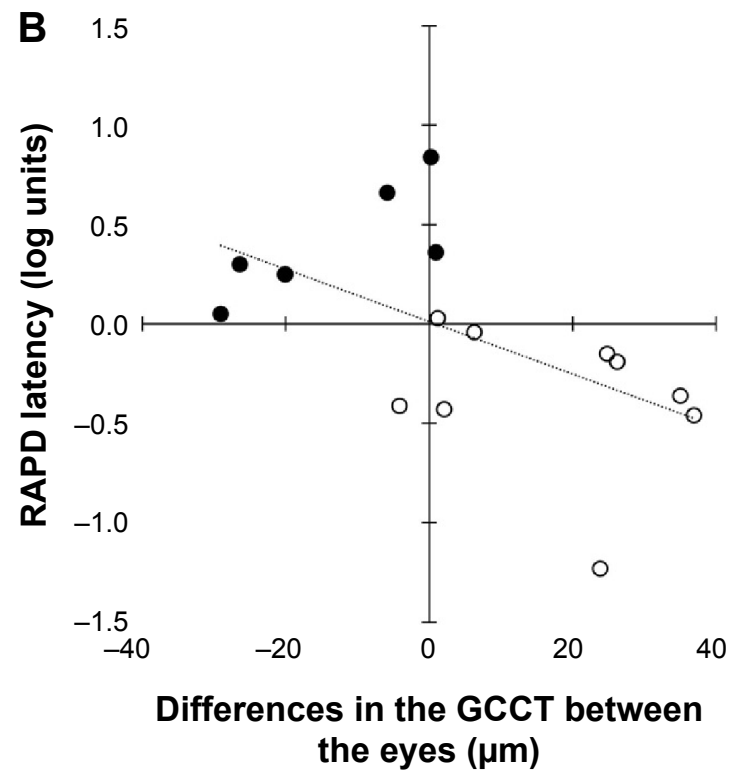

Figure 5 The relationship between the relative afferent pupillary defect (RAPD) and differences in the ganglion cell complex thickness (GCCT).

Notes: Closed circles show patients in whom the right side was affected, and open circles show patients with left optic neuropathy. RAPD amplitude score: $R=-0.593$, $P=0.020$ (A), RAPD latency score: $R=-0.540, P=0.038$ (B). 


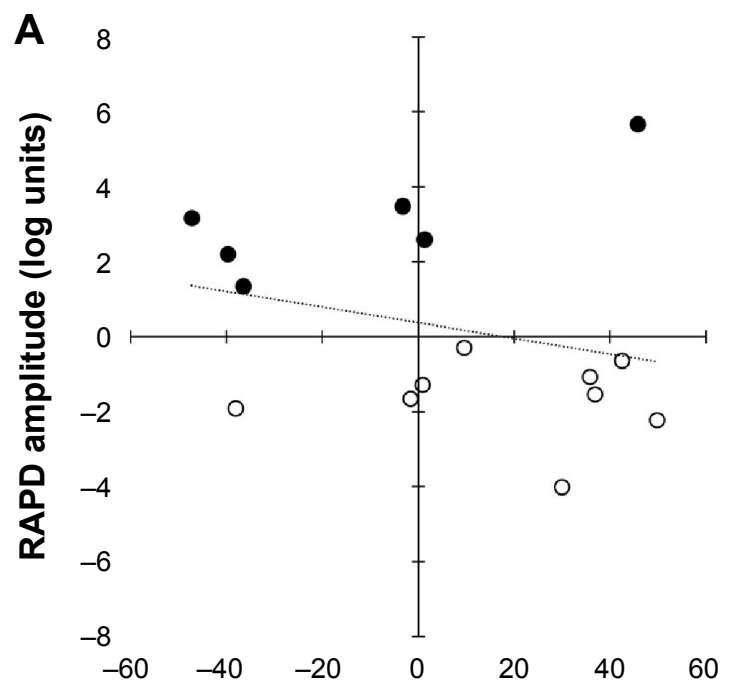

Differences in the cpRNFLT between the eyes $(\mu \mathrm{m})$

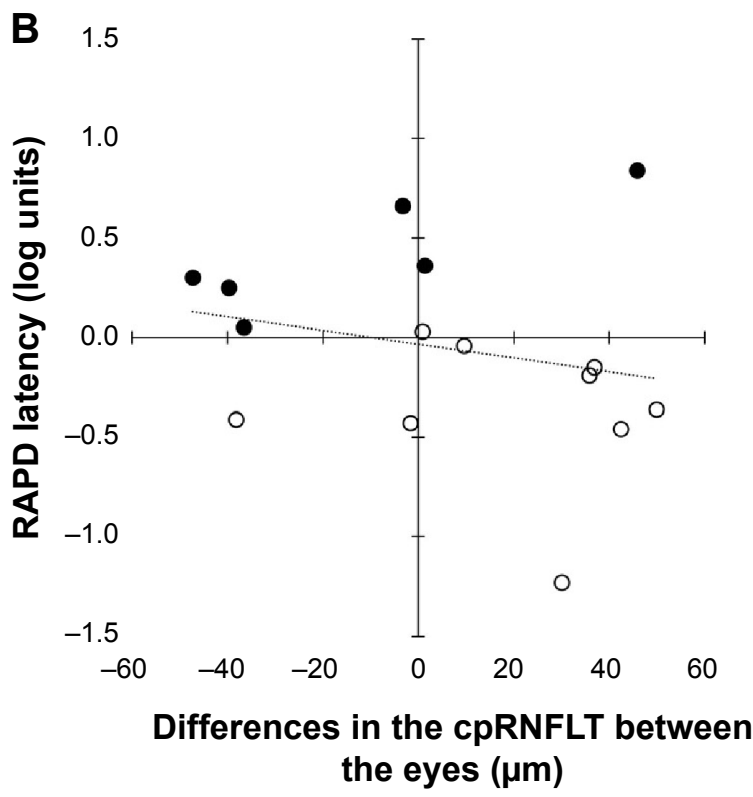

Figure 6 The relationship between the relative afferent pupillary defect (RAPD) and differences in the circumpapillary retinal nerve fiber layer thickness (cPRNFLT). Notes: Closed circles show patients in whom the right side was affected, and open circles show patients with left optic neuropathy. RAPD amplitude score: $R=-0.267$, $P=0.337$ (A), RAPD latency score: $R=-0.228, P=0.413$ (B).

Our results suggest that the RAPD amplitude score is more suitable for detecting optic nerve disease than the RAPD latency score. Therefore, we concentrate on the RAPD amplitude score in the following section when comparing the RAPD with other visual functions or retinal thicknesses.

\section{Relationship between the RAPD amplitude score and the VA of patients with optic nerve disease}

In our study, the VA correlated with the RAPD amplitude score of patients with optic nerve disease. Thompson et al reported that there was no correlation between the RAPD and VA in cases with anterior ischemic optic neuropathy, maculopathy, traumatic optic neuropathy (TON), glaucoma, or retinal vascular disease. ${ }^{12}$ Even in cases of a nerve fiber defect that causes pupil defects, the VA may not be affected in patients with disorders sparing the macula area. In this study, the VA correlated with the RAPD of patients with optic nerve disease, which often causes nerve fiber defects of the macular area. We detected the RAPD of the affected eye in five patients with no or subtle VA laterality. Those five patients were examined for $>1$ year after the onset, and the VA improved over time. In other words, the RAPD remained for a longer time than the VA losses in these patients with optic nerve disease. The correlation between the visual function and RAPD scores appears to depend, at least in part, on the elapsed time after the onset of the disease.

\section{Relationship between the RAPD amplitude score and the central CFF of patients with optic nerve disease}

When there is repeated blinking of a light within a short time, we notice it as a flicker. If the frequency of this light becomes fast, it is noticed as consecutive light, and is indicated as the boundary of the CFF score. The CFF reflects a function of the magnocellular pathway and detects lesions from the retinal ganglion cells (RGCs) to the centrum. ${ }^{21}$

In our study, the central CFF correlated with the RAPD amplitude score of patients with optic nerve disease. Ogasawara et al reported that the CFF correlated with the RAPD of patients with optic nerve disease, glaucoma, and chiasmal syndrome in a study using an ND filter. ${ }^{22}$ Furthermore, they added an ND filter, which was equivalent to the RAPD of the affected eye, over the unaffected eye, remeasured the central CFF, and reported that the central CFF score of both eyes was similar after this change. In other words, both the RAPD and central CFF reflect a difference in the visual input to the eyes. Therefore, it appears reasonable that the central CFF correlated with the RAPD in patients with optic nerve disease causing nerve fiber defects of the macular area.

We detected the RAPD of the affected eye in five patients with small central CFF laterality. Those five patients were examined for $>1$ year after the onset, and the central CFF had already improved at the time of examination. In all patients tested early after the onset, the central CFF was found to be decreased, and the RAPD was detected. Otori et al 
reported that the central CFF decreases earlier than the VA in patients with optic nerve disease due to demyelination and inflammation, ${ }^{21}$ and its recovery occurs later than that of the VA. However, the RAPD was detected in the patients in whom the central CFF had already improved in 1 year or more after the onset. This finding suggests that the RAPD remained for a longer time than the central CFF abnormality in patients with optic nerve disease. These results show that the RAPD may be a more sensitive measure than the central CFF for detecting optic nerve diseases.

\section{Relationship between the RAPD amplitude score and the GCCT of patients with optic nerve disease}

Because half of the RGCs are present in the macula, it is not surprising that macular thinning has been noted using time-domain optical coherence tomography in patients with glaucoma and optic nerve disease. ${ }^{23}$ Recently, the measurement of the retinal inner layer has become possible using SD-OCT, and it has been noted that the ability to detect optic nerve disease by GCC measurements may be equivalent to cpRNFL. To date, there have been no reports comparing the RAPD using pupillography and retinal thickness in optic nerve disease other than glaucoma.

In the present study, the GCCT correlated with the RAPD amplitude score of patients with optic nerve disease. We detected the RAPD of the affected eye in four patients with a small laterality in the GCCT. Among these four patients, three patients (patient nos 2, 7 and 12) were examined early after the onset, and one patient (patient no 10) had MS with thinning of the GCCT of both eyes. Syc et al reported that the ganglion cell layer showed thinning 3 months after the onset of optic neuritis onset. ${ }^{13}$ The GCCT of the three patients we examined early after the onset did not show thinning. The correlation between the retinal thickness and the RAPD scores also appears to depend on the elapsed time after the onset of the disease. The macular retinal thickness was reported to decrease in patients with MS with or without a history of optic neuritis, showing that the ganglion cells of the macular area and the axons are denatured by $\mathrm{MS}^{24}$

Therefore, even though the RAPD of the patient with MS was detected, the GCCT may fail to reveal interocular differences. Kerrison et al observed the manifestations of the RAPD by performing retinal photocoagulation in monkeys. ${ }^{25}$ They found that the RAPD occurred when $25 \%-50 \%$ of the RGCs disappeared histologically. However, we detected the RAPD even in patients who did not show thinning of the GCCT in our study. The VA, central CFF, and RAPD were all detected in patients with optic neuropathy early after the onset, while the thinning of the GCCT was not seen. A lesion of GCC induced by photocoagulation may be essentially different from a lesion of the GCC due to optic nerve disease. The GCCT may therefore not be the ideal parameter to evaluate the degree of disturbance of the RGCs in the acute phase. Instead, these results show that the RAPD can be used to detect optic nerve disease earlier than the GCCT does.

\section{Relationship between the RAPD amplitude score and the cpRNFLT of patients with optic nerve disease}

The retinal nerve fiber layer is comprised of the axons from RGCs. Accordingly, it is possible to evaluate the RGCs indirectly by observing the retinal nerve fiber layer. The cpRNFL in particular reflects the number of nerve fibers through the optic disc edge, and it is thought that the cpRNFL is related to the number of RGCs. Therefore, an evaluation of the cpRNFLT should be useful for the diagnosis of optic nerve diseases.

The cpRNFLT did not correlate with the RAPD scores of patients with optic nerve disease. Nakanishi et al reported that the cpRNFLT correlated with the RAPD in patients who presented with unilateral optic atrophy by ischemic optic neuropathy or TON. ${ }^{16}$ We detected the RAPD of the affected eye in four patients with small laterality in the cpRNFLT. Among these four patients, three (patient nos 4, 7, and 12) were examined early after the onset, and the other (patient no 10) with MS had thinning of the cpRNFLT in both eyes. One of the three patients that we examined early after the onset did not demonstrate thinning of the cpRNFLT. This was presumably due to the same reason why there was also no GCC thinning. The patient with MS was considered to have had a reduced cpRNFLT regardless of the past optic nerve disease, similar to the findings with regard to the GCCT. In terms of the cpRNFLT of one patient with acute papillitis (patient no 2) and one with an unknown time of onset (patient no 6), the affected eye was thicker than the unaffected eye.

According to a previous report, the cpRNFLT was within the normal range in the retrobulbar type cases during the acute phase of optic neuritis, and an elevation of the cpRNFL was seen in cases with papillitis. Thereafter, the cpRNFLT decreased by an average of $20 \mu \mathrm{m}$ compared to the normal value. ${ }^{26}$ In our study, the RAPD amplitude score did not correlate with the cpRNFLT, partly because we included patients with acute papillitis without optic atrophy. Nakanishi et al reported that in patients who presented with unilateral optic atrophy by ischemic optic neuropathy or TON, the cpRNFLT underwent thinning by $23 \%$ in comparison with the unaffected eye when the RAPD became observable. ${ }^{16}$ 
However, the RAPD was detected before the cpRNFLT decreased in this study in patients early after the onset and in patients with papillitis. When the cpRNFLT thickens in cases with papillitis, the cause is widening of the individual nerve fibers. Therefore, just like in GCCT, the cpRNFLT may not necessarily indicate the degree of optic nerve damage. These results suggest that the RAPD detects optic nerve disease earlier than the cpRNFLT, because we were able to detect the RAPD in the patients without thinning of the cpRNFLT.

\section{Conclusion}

We herein demonstrated that the RAPD amplitude score detected pupillary disturbance in all patients with optic nerve disease and that the RAPD score measured using a pupillography device correlated with the difference in the VA, the central CFF, and the GCCT between two eyes. This study showed that the measurement of the RAPD is useful for detecting optic nerve disease, regardless of the stage of disease.

\section{Disclosure}

The authors report no conflicts of interest in this work.

\section{References}

1. Levatin P. Pupillary escape in disease of the retina or optic nerve. Arch Ophthalmol. 1959;62(5):768-779.

2. Stanley JA, Baise GR. The swinging flashlight test to detect minimal optic neuropathy. Arch Ophthalmol. 1968;80(6):769-771.

3. Thompson HS. Afferent pupillary defects. Pupillary findings associated with defects of the afferent arm of the pupillary light reflex arc. $\mathrm{Am}$ J Ophthalmol. 1966;62(5):860-873.

4. Bell RA, Thompson HS. Relative afferent pupillary defect in optic tract hemianopias. Am J Ophthalmol. 1978;85(4):538-540.

5. Prywes AS. Unilateral afferent pupillary defects in asymmetric glaucoma. Arch Ophthalmol. 1976;94(8):1286-1288.

6. Thompson HS, Corbett JJ, Cox TA. How to measure the relative afferent pupillary defect. Surv Ophthalmol. 1981;26(1):39-42.

7. Wilhelm H, Wilhelm B. Clinical applications of pupillography. J Neuroophthalmol. 2003;23(1):42-49.

8. Chang DS, Boland MV, Arora KS, Supakontanasan W, Chen BB, Friedman DS. Symmetry of the pupillary light reflex and its relationship to retinal nerve fiber layer thickness and visual field defect. Invest Ophthalmol Vis Sci. 2013;54(8):5596-5601.

9. Ozeki N, Yuki K, Shiba D, Tsubota K. Pupillographic evaluation of relative afferent pupillary defect in glaucoma patients. Br J Ophthalmol. 2013;97(12):1538-1542.

10. Tatham AJ, Meira-Freitas D, Weinreb RN, Zangwill LM, Medeiros FA Detecting glaucoma using automated pupillography. Ophthalmology. 2014;121(6):1185-1193.

Clinical Ophthalmology

\section{Publish your work in this journal}

Clinical Ophthalmology is an international, peer-reviewed journal covering all subspecialties within ophthalmology. Key topics include: Optometry; Visual science; Pharmacology and drug therapy in eye diseases; Basic Sciences; Primary and Secondary eye care; Patient Safety and Quality of Care Improvements. This journal is indexed on
11. Sarezky D, Krupin T, Cohen A, Stewart CW, Volpe NJ, Tanna AP Correlation between intereye difference in visual field mean deviation values and relative afferent pupillary response as measured by an automated pupillometer in subjects with glaucoma. J Glaucoma. 2014; 23(7):419-423.

12. Thompson HS, Montague P, Cox TA, Corbett JJ. The relationship between visual acuity, pupillary defect, and visual field loss. Am J Ophthalmol. 1982;93(6):681-688.

13. Syc SB, Saidha S, Newsome SD, et al. Optical coherence tomography segmentation reveals ganglion cell layer pathology after optic neuritis. Brain. 2012;135(pt 2):521-533.

14. Chew SS, Cunnningham WJ, Gamble GD, Danesh-Meyer HV. Retinal nerve fiber layer loss in glaucoma patients with a relative afferent pupillary defect. Invest Ophthalmol Vis Sci. 2010;51(10):5049-5053.

15. Tatsumi Y, Nakamura M, Fujioka M, et al. Quantification of retinal nerve fiber layer thickness reduction associated with a relative afferent pupillary defect in asymmetric glaucoma. Br J Ophthalmol. 2007; 91(5):633-637.

16. Nakanishi Y, Nakamura M, Tatsumi Y, Nagai-Kusuhara A, Negi A. Quantification of retinal nerve fiber layer thickness reduction associated with a relative afferent pupillary defect. Graefes Arch Clin Exp Ophthalmol. 2006;244(11):1480-1484.

17. Lowenstein O. Clinical pupillary symptoms in lesions of the optic nerve, optic chiasm, and optic tract. AMA Arch Ophthalmol. 1954;52(3): 385-403.

18. Alexandridis E, Argyropoulos T, Krastel H. The latent period of the pupil light reflex in lesions of the optic nerve. Ophthalmologica. 1981; 182(4):211-217.

19. Ellis CJ. The afferent pupillary defect in acute optic neuritis. $J$ Neurol Neurosurg Psychiatry. 1979;42(11):1008-1017.

20. Chang DS, Arora KS, Boland MV, Supakontanasan W, Friedman DS. Development and validation of an associative model for the detection of glaucoma using pupillography. Am J Ophthalmol. 2013;156(6): $1285-1296$.

21. Otori T, Nakao Y, Toma N, Manabe R. Clinical evaluation of usefulness of determination of central critical fusion frequency in ocular diseases. Rinsho Ganka. 1973;27:301-310.

22. Ogasawara K, Takahashi Y, Odashima S, Tazawa Y. Re-evaluation of swinging flashlight test in measuring relative afferent pupillary defect. Rinsho Ganka. 1985;39:745-750.

23. Kusuhara S, Nakamura M, Nagai-Kusuhara A, Nakanishi Y, Kanamori A, Negi A. Macular thickness reduction in eyes with unilateral optic atrophy detected with optical coherence tomography. Eye. 2006;20(8): 882-887.

24. Burkholder BM, Osborne B, Loguidice MJ, et al. Macular volume determined by optical coherence tomography as a measure of neuronal loss in multiple sclerosis. Arch Neurol. 2009;66(11):1366-1372.

25. Kerrison JB, Buchanan K, Rosenberg ML, et al. Quantification of optic nerve axon loss associated with a relative afferent pupillary defect in the monkey. Arch Ophthalmol. 2001;119(9):1333-1341.

26. Costello F, Coupland S, Hodge W, et al. Quantifying axonal loss after optic neuritis with optical coherence tomography. Ann Neurol. 2006;59(6): 963-969.

\section{Dovepress}

PubMed Central and CAS, and is the official journal of The Society of Clinical Ophthalmology (SCO). The manuscript management system is completely online and includes a very quick and fair peer-review system, which is all easy to use. Visit http://www.dovepress.com/ testimonials.php to read real quotes from published authors. 